\title{
Prevalence of arterial hypertension in patients with atrial fibrillation undergoing ablation - a prospective, cohort study
}

\author{
Anna E. Platek, Filip M. Szymanski, Krzysztof J. Filipiak, Anna Rys, Marcin Kotkowski, \\ Karolina Semczuk, Grzegorz Opolski
}

$1^{\text {st }}$ Department of Cardiology, Medical University of Warsaw, Warsaw, Poland

\begin{abstract}
Summary
Background Arterial hypertension is one of the major cofounders in the development of atrial fibrillation (AF) and hypertension is commonly found in AF patients. The aim of the study was to establish the prevalence of arterial hypertension in patients undergoing ablation, who are relatively young and healthy group of AF patients.

Material and methods 266 consecutive patients admitted for AF ablation were screened for arterial hypertension. All patients had their blood pressure measured on admission by, prior to the ablation procedure by a qualified physician, according to the current guidelines. Also, medical records of patients were reviewed for the previous diagnosis of hypertension or taking hypotensive agents.

Results The study group was predominantly male (65.0\%; mean age $57.6 \pm 10.1$ years). Mean body mass index was $29.7 \pm 5.0 \mathrm{~kg} / \mathrm{m}^{2}$. Paroxysmal AF was present in $69.5 \%$ of patients. In $72.9 \%$ of patients hypertension was diagnosed previously. On admission, mean systolic and diastolic blood pressure values were $131.7 \pm 16.7$ and 80.7 $\pm 11.1 \mathrm{~mm} \mathrm{Hg.} 123(46.2 \%)$ patients had systolic and/or diastolic blood pressure values respectively $>140 \mathrm{and} /$ lor $>90 \mathrm{~mm} \mathrm{Hg}$. Patients with previously diagnosed hypertension were older $(58.7 \pm 8.7$ vs $54.6 \pm 12.7$ years; $\mathrm{p}=0.003)$, had higher BMI $\left(30.3 \pm 5.0\right.$ vs $\left.28.1 \pm 4.8 \mathrm{~kg} / \mathrm{m}^{2} ; \mathrm{p}=0.002\right)$, and more often history of diabetes $(10.8 \%$ vs $1.4 \% ; \mathrm{p}=0.03)$ compared to non-hypertensive group. There were no differences in terms of history of dyslipidaemia, stroke, myocardial infarction or family history of cardiovascular disease.

Conclusions In patients with AF undergoing ablation procedure, prevalence of diagnosed arterial hypertension is very high, much higher than in the general population. Nevertheless, majority of patients meet the criteria for adequate blood pressure control.

key words: arterial hypertension, atrial fibrillation, ablation
\end{abstract}

Arterial Hypertens. 2015, vol. 19, no. 3, pages: 135-139

DOI: $10.5603 / A H .2015 .0017$

\section{Background}

Arterial hypertension and atrial fibrillation (AF) are one of the most prevalent diseases of the cardiovascular system. The incidence of both conditions increases with advancing age [1]. Therefore, due to the prolonging life expectancy, the total number of patients with arterial hypertension and $\mathrm{AF}$ is expected to grow in the forthcoming years. Both $\mathrm{AF}$ and arterial hypertension are responsible for considerable morbidity and mortality. Their coexistence further increases the risk and affects management of patients

Address for correspondence: Filip M. Szymanski, MD, PhD 1st Department of Cardiology, Medical University of Warsaw 1 A Banacha Street, 02-097 Warsaw, Poland

tel.: +48 22 599-19-58; fax: +48 22 599-19-57; e-mail address: filip.szymanski@wum.edu.pl 
and treatment strategies. Currently, the treatment of arterial hypertension depends mostly on lifestyle modifications and pharmacotherapy, with invasive treatment still playing only a minor role in everyday practice [2]. Whereas, the treatment of AF, namely pursing the rhythm-control strategy, in many cases is achieved through catheter ablation. Current guidelines recommend ablation as one of the treatment options in patients with symptomatic $\mathrm{AF}$ in spite of an antiarrhythmic drug therapy or as first-line or second line therapy in selected patients, which prefer this kind of treatment [3, 4]. Unfortunately, effectiveness of AF ablations remains limited, partially due to poor control of risk factors for the arrhythmia recurrence, which include diabetes, metabolic syndrome, or left atrial dimensions [5]. Arterial hypertension contributes to the development of AF itself, as well as the established AF-recurrence predictors. Therefore, screening for arterial hypertension in $\mathrm{AF}$ patients and describing the profile of hypertensive patients, especially those undergoing ablation is of great importance.

\section{Material and methods}

We performed a prospective, observational, cohort study in order to establish the prevalence of arterial hypertension in consecutive Polish patients undergoing catheter ablation for AF. The study was conducted with respect to the Declaration of Helsinki. The study protocol was approved by the Ethics Committee by the Medical University of Warsaw and a written, informed consent was obtained from every study participant prior to the study enrolment.

The study cohort consisted of 266 consecutive patients who were hospitalized in a high-volume tertiary university cardiology clinic, where they were admitted for first-ever ablation for AF. All patients were qualified for the ablation procedure following the European Society of Cardiology (ESC) guidelines for the management of AF from 2010 and 2012 [3, 4]. The study protocol was described in detail previously $[5,6]$. Exclusion criteria were age $<18$ or $\geq 75$ years, prior ablation of AF, MI, stroke or decompensation of heart failure within the last six months, untreated overt hyper- or hypothyroidism, current treatment with a continuous positive airway pressure device, and absence of written informed consent.

AF diagnosis was made based on at least one episode of the AF recorded in a 12-lead electrocardiogram (ECG) or an episode of documented AF in a 12-lead ECG and/or 24-hour ECG Holter monitoring in 6 months prior to the study enrolment.
AF was defined as at least 30 seconds of an irregular heart rhythm with no distinct $P$ waves, and irregular $\mathrm{RR}$ intervals. In all cases the diagnosis was made by two independent expert cardiologists. Paroxysmal $\mathrm{AF}$ was defined as a self-terminating episode, whereas persistent $\mathrm{AF}$ was diagnosed when arrhythmia episode either lasted longer than 7 days or required termination by cardioversion. There were no cases of permanent $\mathrm{AF}$ in the study population because this diagnosis is made only if it was decided not to pursue rhythm control strategy.

All study participants were screened for arterial hypertension. Patients had their blood pressure measured on admission, prior to the ablation procedure, by a qualified physician Diagnosis of arterial hypertension was made when office blood pressure were equal or exceeded the values of 140 for systolic blood pressure (SBP) and/or 90 for diastolic blood pressure (DBP). The number of required measurements, measure techniques and settings were in accordance with the current guidelines for arterial hypertension diagnosis and management of the ESC and European Society of Hypertension [2]. The diagnosis was also made were there were eligible medical records available on previous diagnosis of arterial hypertension or taking prescription hypotensive agents.

All patients were also screened for other classical and non-classical cardiovascular risk factors including diabetes mellitus, dyslipidaemia, or history of cardiovascular disease. Diagnosis of all factors was made based on eligible medical records, taking prescription drugs applicable for the respective disease (i.e. hypoglycaemic agents for diabetes), or as a de novo diagnosis according to the current diagnostic criteria.

Statistical analysis was performed using the SAS software version 8.02 (SAS Institute, Inc., Cary, NC, USA). Continuous data are presented as mean \pm standard deviation (SD) and were compared using the Mann-Whitney test or Student's t-test. Categorical variables were compared using either the $\chi^{2}$ or Fisher exact tests. A p value of less than 0.05 was considered statistically significant, whereas the confidence intervals (CI) were 95\%.

\section{Results}

This prospective cohort study covered 266 patients undergoing first-ever ablation for AF. The mean age of the study population was $57.6 \pm 10.1$ years and $65 \%$ of patients were male. The body mass index (BMI) in the study population was $29.7 \pm 5.0 \mathrm{~kg} / \mathrm{m}^{2}$ and $69.5 \%$ of patients had paroxysmal AF, while 
Table 1. Baseline characteristics of the study population

\begin{tabular}{|c|c|}
\hline Parameter & $\begin{array}{c}\text { Value } \\
n=266\end{array}$ \\
\hline Age (years) & $57.6 \pm 10.1$ \\
\hline BMI $\left[\mathrm{kg} / \mathrm{m}^{2}\right]$ & $29.7 \pm 5.0$ \\
\hline $\mathrm{SBP}[\mathrm{mm} \mathrm{Hg}]$ & $131.7 \pm 16.7$ \\
\hline $\mathrm{DBP}[\mathrm{mm} \mathrm{Hg}]$ & $80.7 \pm 11.0$ \\
\hline HR (beats per minute) & $75.0 \pm 15.8$ \\
\hline History of myocardial infarction & $22(8.3 \%)$ \\
\hline Diabetes mellitus & $17(6.4 \%)$ \\
\hline Previously diagnosed arterial hypertension & $194(72.9 \%)$ \\
\hline Smoking & $28(10.5 \%)$ \\
\hline History of stroke or TIA & $22(8.3 \%)$ \\
\hline Dyslipidaemia & $167(62.8 \%)$ \\
\hline Thyroid disease & $68(25.6 \%)$ \\
\hline Family history of cardiovascular disease & $104(39.0 \%)$ \\
\hline Paroxysmal AF & $185(69.5 \%)$ \\
\hline
\end{tabular}

the rest was diagnosed with persistent AF. Detailed characteristics of the study population are listed in the Table 1.

As for the mean SBP and DBP values they were $131.7 \pm 16.7$ and $80.7 \pm 11.0 \mathrm{~mm} \mathrm{Hg}$, respectively (Figure 1). One hundred ninety-four patients $(72.9 \%)$ were previously diagnosed with arterial hypertension, while the values exceeding the threshold of $\geq 140$ and/or $90 \mathrm{~mm} \mathrm{Hg}$ on admission were found in $46.2 \%$ of patients. No data were available in the medical records on the degree of the previously diagnosed hypertension prior to the implemented treatment.

After dividing patients according to the presence of previously diagnosed arterial hypertension it was found that patients with the disease were older $(58.7 \pm 8.7$ vs $54.6 \pm 12.7$ years; $\mathrm{p}=0.003)$, and had higher BMI $\left(30.3 \pm 5\right.$ vs $28.1 \pm 4.8 \mathrm{~kg} / \mathrm{m}^{2}$; $\mathrm{p}=0.002)$ than patients without the diagnosed arterial hypertension. As for the prevalence of comorbidities it was found that hypertensive patients more often had diabetes mellitus ( $10.8 \%$ vs $1.4 \% ; \mathrm{p}=0.03$ ) compared with patients without the diagnosis. SBP values were significantly higher in patients with previously diagnosed arterial hypertension (133.6 \pm 16.1 vs $126.7 \pm 17.6 \mathrm{~mm} \mathrm{Hg}$; $=0.003)$ than those without the disease. No differences between the groups were found in terms of DBP $(81.4 \pm 11.4$ vs $78.9 \pm 10.1 \mathrm{~mm} \mathrm{Hg} ; \mathrm{p}=0.1$ ).

No differences were seen in terms of the prevalence of other cardiovascular risk factors including:

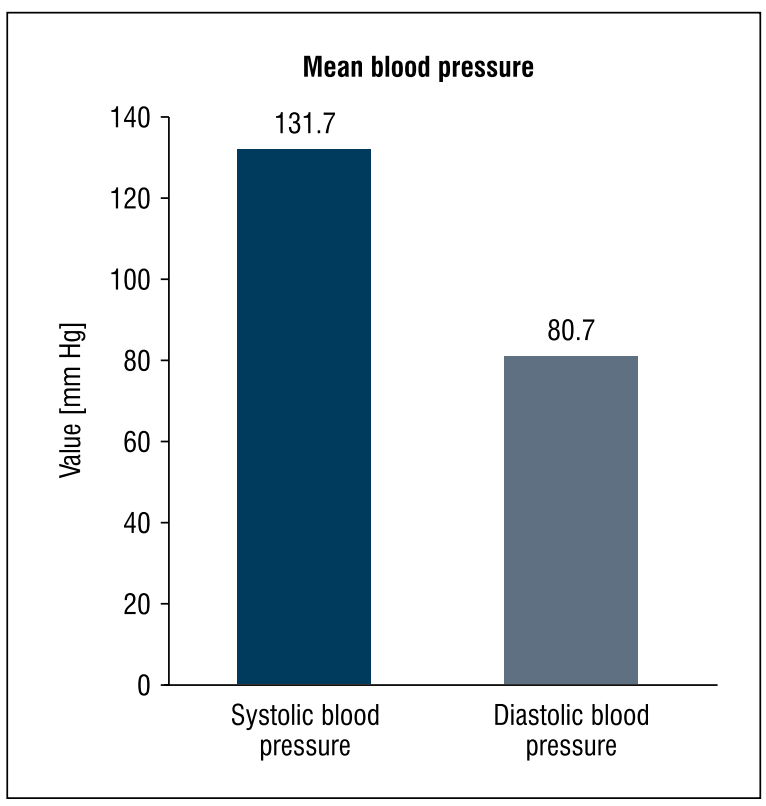

Figure 1. Mean blood pressure on admission

history of myocardial infarction or stroke, dyslipidaemia, smoking and family history of cardiovascular disease $(p>0.05)$. The detailed information on the groups characteristics are presented in Table 2.

\section{Discussion}

The present study concerned patients undergoing ablation for AF. This group is somehow different from the general population of AF patients due to a number of factors. While there is no strict age limit for the catheter ablation procedure, it is rarely performed in patients older than 75 years [8]. Moreover, number of contraindications for the procedure contributes to lower number of comorbidities in $\mathrm{AF}$ ablation recipients. Therefore, compared to the unselected groups of AF patients, those undergoing ablation procedure tend to be in better general health and have lower comorbidity rate. As it was shown in the present study, the mean age of the participants was 57.6 years, whereas in the AF general population it tends to be over 70 years [9]. Nevertheless, the prevalence of arterial hypertension in the present group was high $(72.9 \%)$, almost twice as high as in the corresponding age group of the general population [10]. Higher prevalence of the disease is attributable to two factors. First of all, prior to the ablation all patients were screened and treated by specialists therefore the rate of underdiagnosed cases of arterial hypertension remains low in this population. Second of all, there is a strong relationship between hypertension and development of $\mathrm{AF}$. 
Table 2. Patients' characteristics according to the presence of previously diagnosed hypertension.

\begin{tabular}{l|c|c|c}
\hline Parameter & $\begin{array}{c}\text { Patients with previously diagnosed hypertension } \\
(\mathbf{n}=194)\end{array}$ & $\begin{array}{c}\text { Non-hypertensive patients } \\
\text { (n= 72) }\end{array}$ & p value \\
\hline Age (years) & $58.7 \pm 8.7$ & $54.6 \pm 12.7$ & $\mathbf{0 . 0 0 3}$ \\
\hline $\mathrm{BMI}\left[\mathrm{kg} / \mathrm{m}^{2}\right]$ & $30.3 \pm 5$ & $28.1 \pm 4.8$ & $\mathbf{0 . 0 0 2}$ \\
\hline $\mathrm{SBP}[\mathrm{mm} \mathrm{Hg}]$ & $133.6 \pm 16.1$ & $126.7 \pm 17.6$ & $\mathbf{0 . 0 0 3}$ \\
\hline DBP $[\mathrm{mm} \mathrm{Hg]}$ & $81.4 \pm 11.4$ & $78.9 \pm 10.1$ & 0.1 \\
\hline HR (beats per minute) & $76.4 \pm 16.5$ & $71.1 \pm 13.2$ & 0.06 \\
\hline History of myocardial infarction & $18(9.3 \%)$ & $4(5.6 \%)$ & 0.46 \\
\hline Diabetes mellitus & $21(10.8 \%)$ & $1(1.4 \%)$ & $\mathbf{0 . 0 3}$ \\
\hline Smoking & $20(10.3 \%)$ & $8(11.1 \%)$ & 0.85 \\
\hline History of stroke or TIA & $18(9.3 \%)$ & $4(5.6 \%)$ & 0.47 \\
\hline Dyslipidaemia & $124(63.9 \%)$ & $43(59.7 \%)$ & 0.62 \\
\hline Thyroid disease & $52(26.8 \%)$ & $16(22.2 \%)$ & 0.45 \\
\hline Family history of cardiovascular disease & $77(39.7 \%)$ & $27(37.5 \%)$ & 0.89 \\
\hline Paroxysmal AF & $129(66.5 \%)$ & $56(77.8 \%)$ & 0.08 \\
\hline
\end{tabular}

Parameters are presented as mean \pm standard deviation or $n(\%)$

$\mathrm{BMI}$ - body mass index, SBP — systolic blood pressure, DBP — diastolic blood pressure, HR — heart rate, TIA — transient ischaemic attack, AF — atrial fibrillation

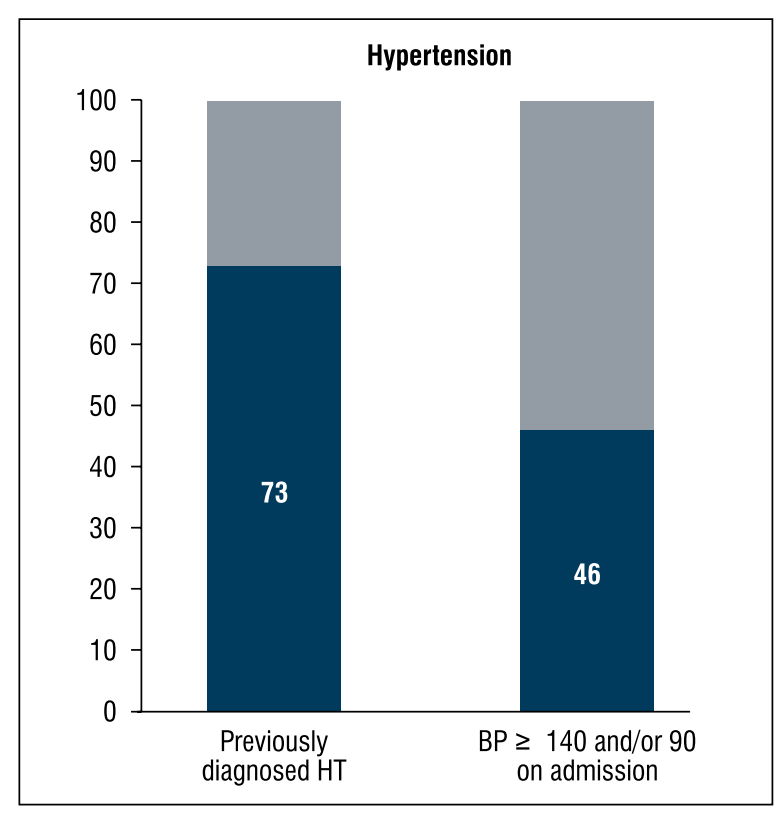

Figure 2. Prevalence of previously diagnosed arterial hypertension versus patients with blood pressure values meeting the criteria for hypertension diagnosis

BP - blood pressure, HT - arterial hypertension

Arterial hypertension is a disease associated with haemodynamic changes in the whole cardiovascular system, which lead to changes and damage to the end-organs. Cohort epidemiological studies of hypertensive patients show that those who subsequently developed AF had higher mean systolic blood pressure values than those who maintained the sinus rhythm [11]. In other studies the prevalence of ar- terial hypertension in AF patients was higher than $50 \%$, and it was thought to be a sole cause of the $\mathrm{AF}$ in $15 \%$ of patients [12-14]. The proposed mechanism in which arterial hypertension causes the $\mathrm{AF}$ involves the structural changes in the left atrium that include left atrial stretch and enlargement, changes in left atrial mechanical function, altered left atrial electrophysiology, and increased atrial ectopic activity. Mostly, due to haemodynamic overload [15].

Other contributors to the AF development that were described in the literature involve advanced age, obesity, smoking, diabetes mellitus, thyroid dysfunction, or history of diseases such as heart valve disease, heart failure, myocardial infarction [16]. In the present study patients with previously diagnosed arterial hypertension were older, had higher BMI and higher prevalence of diabetes mellitus. As they are all contributors to the development of arrhythmia itself, there is a possibility that in this cohort, the hypertensive patients will be predisposed to higher risk of transition to the permanent form of the arrhythmia in the future or lower success rate after the ablation procedure.

Data on the impact of hypertension itself on the ablation procedure vary across the literature. Studies focused on establishing the parameters predictive of AF recurrence after the procedure do not identify arterial hypertension as one of the risk factors [17] or even list hypertension as one of the procedure success predictors [18]. This may be partially attributable to the fact that most of the AF ablation recipients with hypertension receive hypotensive treatment on 
hospital discharge and the reduction of blood pressure values both pharmacological and invasive are associated with improvement of the success rate [19, 20]. Moreover, the successful procedure itself was described to be associated with a significant decrease in the systolic blood pressure values [21]. As the treatment of hypertension may reverse the structural changes in the heart, the optimistic finding of the present study was the in spite of high arterial hypertension prevalence, the mean SBP and DBP values were relatively low, which points to a good blood pressure control in this cohort.

\section{Conclusions}

Patients with AF undergoing ablation are a relatively young group, with a low comorbidity rate. Nevertheless, in this group of patients, prevalence of diagnosed arterial hypertension is very high, much higher than in the age corresponding group from the general population. Patients with diagnosed hypertension tend to be older, have higher BMI, and more often history of diabetes. Nevertheless, the mean SBP and DBP values on admission were not significantly elevated, which shows that the majority of patients meet the criteria for adequate blood pressure control.

\section{References}

1. Healey J.S., Connolly S.J. Atrial fibrillation: hypertension as a causative agent, risk factor for complications, and potential therapeutic target. Am. J. Cardiol. 2003; 91: 9G-14G.

2. Mancia G., Fagard R., Narkiewicz K. et al. 2013 ESH/ESC guidelines for the management of arterial hypertension: the Task Force for the Management of Arterial Hypertension of the European Society of Hypertension (ESH) and of the European Society of Cardiology (ESC). Eur. Heart J. 2013; 34: 2159-2219.

3. Camm A.J., Kirchhof P., Lip G.Y. et al. European Heart Rhythm Association; European Association for Cardio-Thoracic Surgery. Guidelines for the management of atrial fibrillation: the Task Force for the Management of Atrial Fibrillation of the European Society of Cardiology (ESC). Eur. Heart J. 2010; 31: 2369-2429.

4. Camm A.J., Lip G.Y., De Caterina R. et al. ESC Committee for Practice Guidelines (CPG). 2012 focused update of the ESC Guidelines for the management of atrial fibrillation: an update of the 2010 ESC Guidelines for the management of atrial fibrillation. Developed with the special contribution of the European Heart Rhythm Association. Eur. Heart J. 2012; 33: 2719-2147.

5. Cai L., Yin Y., Ling Z. et al. Predictors of late recurrence of atrial fibrillation after catheter ablation. Int. J. Cardiol. 2013; 164: 82-87.
6. Szymański F.M., Płatek A.E., Karpiński G., Koźluk E., Puchalski B., Filipiak K.J. Obstructive sleep apnoea in patients with atrial fibrillation: prevalence, determinants and clinical characteristics of patients in Polish population. Kardiol. Pol. 2014; 72: 716-724.

7. Szymanski F.M., Filipiak K.J., Platek A.E. et al. Presence and severity of obstructive sleep apnea and remote outcomes of atrial fibrillation ablations - a long-term prospective, cross-sectional cohort study. Sleep Breath. 2015 Jan 8. doi: 10.1007/s11325-014-1102-x [Epub ahead of print].

8. Chen J., Hocini M., Larsen T.B., Proclemer A., Sciaraffia E., Blomström-Lundqvist C. Scientific Initiative Committee, European Heart Rhythm Association. Clinical management of arrhythmias in elderly patients: results of the European Heart Rhythm Association survey. Europace 2015; 17: 314-317.

9. Hrynkiewicz-Szymanska A., Dluzniewski M., Platek A.E. et al. Association of the CHADS2 and CHA2DS2-VASc scores with left atrial enlargement: a prospective cohort study of unselected atrial fibrillation patients. J. Thromb. Thrombolysis 2015; 40: 240-247.

10. Zdrojewski T., Bandosz P., Rutkowski M. et al. Rozpowszechnienie, wykrywanie i skuteczność leczenia nadciśnienia tętniczego w Polsce — wyniki badania NATPOL 2011. Nadciśnienie Tętnicze 2014; 18: 116-117.

11. Ciaroni S., Cuenoud L., Bloch A. Clinical study to investigate the predictive parameters for the onset of atrial fibrillation in patients with essential hypertension. Am. Heart J. 2000; 139: 814-819.

12. Kannel W.B., Wolf P.A., Benjamin E.J., Levy D. Prevalence, incidence, prognosis, and predisposing conditions for atrial fibrillation: population-based estimates. Am. J. Cardiol. 1998; 82 (suppl.): $2 \mathrm{~N}-9 \mathrm{~N}$.

13. Kannel W.B., Abbott R.D., Savage D.D., McNamara P.M. Epidemiologic features of chronic atrial fibrillation: the Framingham study. N. Engl. J. Med. 1982; 306: 1018-1022.

14. Krahn A.D., Manfreda J., Tate R.B., Mathewson F.A., Cuddy T.E. The natural history of atrial fibrillation: incidence, risk factors, and prognosis in the Manitoba Follow-Up Study. Am. J. Med. 1995; 98: 476-484.

15. Healey J.S., Connolly S.J. Atrial fibrillation: hypertension as a causative agent, risk factor for complications, and potential therapeutic target. Am. J. Cardiol. 2003; 91(10A): 9G-14G.

16. Andrade J., Khairy P., Dobrev D., Nattel S. The clinical profile and pathophysiology of atrial fibrillation: relationships among clinical features, epidemiology, and mechanisms. Circ. Res. 2014; 114: 1453-1468.

17. Chodór P., Białkowska B., Hapeta B. et al. Evaluation of factors affecting persistence of atrial fibrillation in patients with concomitant atrial flutter treated with percutaneous radiofrequency current ablation of the right atrial cavotricuspid isthmus. Kardiol. Pol. 2013; 71: $247-252$.

18. Heist E.K., Chalhoub F., Barrett C., Danik S., Ruskin J.N., Mansour M. Predictors of atrial fibrillation termination and clinical success of catheter ablation of persistent atrial fibrillation. Am. J. Cardiol. 2012; 110: 545-551.

19. Mohanty S., Mohanty P., Trivedi C. et al. Association of pretreatment with angiotensin-converting enzyme inhibitors with improvement in ablation outcome in atrial fibrillation patients with low left ventricular ejection fraction. Heart Rhythm 2015 Jun 5. pii: S1547-5271(15)00697-9. doi: 10.1016/j.hrthm.2015.06.007. [Epub ahead of print].

20. Pokushalov E., Romanov A., Katritsis D.G. et al. Renal denervation for improving outcomes of catheter ablation in patients with atrial fibrillation and hypertension: early experience. Heart Rhythm 2014; 11: 1131-1138. 